\title{
Paraneoplastic syndrome and underlying breast cancer: a worsening rash despite initiation of chemotherapy
}

\author{
Shradha Ahuja, MD, ${ }^{a}$ Priyanka Makkar, MD, ${ }^{a}$ Sorab Gupta, MD, ${ }^{a}$ and Ivette Vigoda, $\mathrm{MD}^{\mathrm{b}}$
}

Departments of ${ }^{a}$ Internal Medicine and ${ }^{b}$ Hematology-Oncology, Saint Barnabas Hospital, Bronx, New York

\begin{abstract}
kin may show the first clinical evidence of sysS temic disease and can be the first clue to malignancy in $1 \%$ of cases. ${ }^{1}$ Dermatomyositis is an immunologically mediated inflammatory myopathy characterized by proximal muscle weakness, muscle inflammation, and characteristic skin findings. ${ }^{2}$ It has an incidence of 1 in 100,000 patients. ${ }^{3}$ In 15\%$30 \%$ cases of dermatomyositis, an underlying malignancy is the cause of paraneoplastic syndrome. ${ }^{4,5}$ Ovarian and breast cancer in women and lung cancer in men are the most common malignancies associated with dermatomyositis. ${ }^{6}$ Here we report the case of a 55-year-old postmenopausal woman who initially presented with a facial rash. She was treated for chemical dermatitis without resolution of symptoms and was subsequently found to have dermatomyositis associated with stage IV invasive ductal carcinoma of the breast. In most cases, the skin changes resolve after treatment for the underlying malignancy has been initiated, but in this case of paraneoplastic dermatomyositis, the rash worsened with initiation of treatment for underlying breast cancer.
\end{abstract}

\section{Case presentation and summary}

The woman presented to the emergency department (ED) with a 5-day history of a pruritic rash on her face and chest. She had emigrated from Peru to the Unites States 5 years previously. Her family history was significant for breast cancer in her sister (diagnosed at age 29 years), colon cancer in her paternal half-sister (diagnosed, age 20), and ovarian cancer in her paternal aunt (diagnosed, age 40). On exam she was found to have a violaceous, nonblanching rash over her face (Figure 1), involving the cheeks, nose, forehead, chin, chest anteriorly, upper back, and arms posteriorly, with areas of hyperpigmentation. The rash was diagnosed as photocontact dermatitis, and she was discharged home on oral steroids.

The woman returned to the $\mathrm{ED}$ a few days later with no improvement in the rash and mentioned that she had detected a lump on her left breast. She also complained of fatigue and weakness in her proximal arms and legs. Physical examination revealed a nontender, hard, fixed mass of $3 \times 3 \mathrm{~cm}$ in the inner outer quadrant of her left breast without overlying skin changes. Serological tests showed elevated levels of alanine transaminase (ALT; $44 \mathrm{U} / \mathrm{L}$; normal, 0-35 U/L), aspartate aminotransferase (AST; 83 $\mathrm{U} / \mathrm{L}$; normal, 0-35 U/L), creatine kinase (838 U/L; normal, 30-170 U/L), and aldolase (5 U/L; normal, $<7.5 \mathrm{U} / \mathrm{L})$. She was ANA-positive with a titer of 1:160 and her anti-Jo-1 serum levels were normal.

The patient was referred to the hospital's breast oncology department and then the dermatology department. A biopsy as per oncology recommendations revealed a poorly differentiated invasive ductal carcinoma (ER-negative, PR-negative, HER2neu-negative). The results of a positron-emission tomography-computed-tomography (PET-CT) scan revealed increased uptake in 2 axillary, 1 internal mammary, and 1 anterior mediastinal lymph nodes. A video-assisted thoracoscopic surgical lymph node biopsy of the mediastinal node revealed metastatic disease. Genetic testing was positive for BRCA1-3071-del T (deleterious) mutation. Given the clinical features with concurrent diagnosis of breast malignancy, a skin biopsy was obtained from the back to rule out paraneoplastic dermatomyositis as per dermatology recommendations. Histological findings on the skin punch biopsy were consistent with dermatomyositis with concordant vacuolar interface dermatitis (Figure 2).

The patient was put on carboplatin $2 \mathrm{mg}$ and gemcitabine $1,000 \mathrm{mg} / \mathrm{m}^{2}$ and received 7 cycles. We consulted our colleagues in the rheumatol-

Accepted for publication August 26, 2015. Correspondence: Sorab Gupta, MD; drsorabgupta@gmail.com. Disclosures: The authors report no disclosures or conflicts of interest. JCSO 2016;14:229-231. (2016 Frontline Medical Communications. doi: 10.12788 /jcso.0186. 


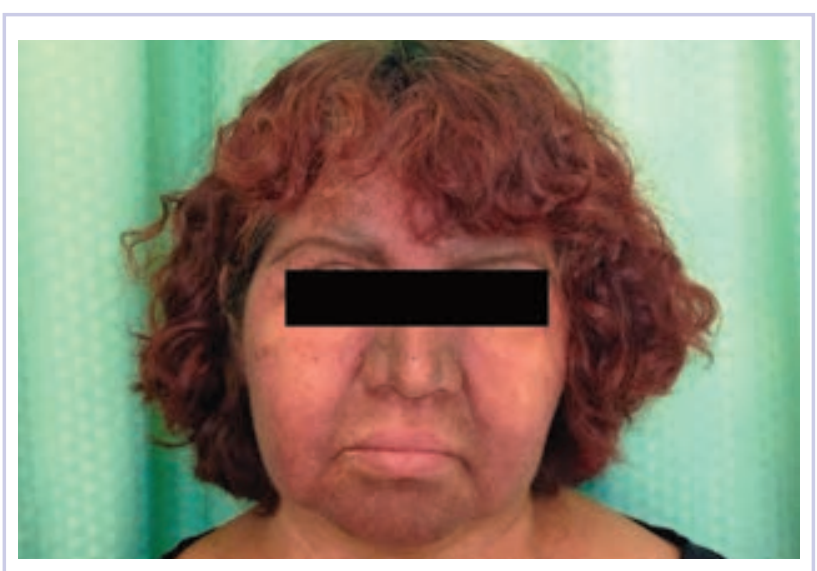

FIGURE 1 Violaceous rash on face and cheek.

ogy department about the patient's rash, and they put her on oral and topical steroids as well as biweekly injections of adrenocorticotropic hormone (ACTH) for 12 weeks. This combination of medication led to improvement in the rash.

After 5 months of chemotherapy, another PET-CT scan was done and showed a stable disease with no improvement. The patient was switched to CMF (carboplatin 2 $\mathrm{mg}$, methotrexate $40 \mathrm{mg} / \mathrm{m}^{2}$, and fluorouracil $600 \mathrm{mg} /$ $\mathrm{m}^{2}$ ) but received only 1 cycle before treatment was again switched, this time to carboplatin $2 \mathrm{mg}$ and paclitaxel 80 $\mathrm{mg} / \mathrm{m}^{2}$ because of a possible benefit as per the literature. ${ }^{7}$ However, that regimen was discontinued after 4 cycles because of the side effects, mainly fatigue. The rash deteriorated with increased hyperpigmentation with the changed chemotherapy regimen. The PET-CT scan was repeated after the end of the 4th cycle and compared with the scan that had been done after the first chemotherapy regimen. The radiologist notified us that the first scan had been read incorrectly and had in fact shown complete response to the chemotherapy regimen. The initial chemotherapy regimen of carboplatin $2 \mathrm{mg}$ and gemcitabine $1,000 \mathrm{mg} /$ $\mathrm{m}^{2}$ was resumed after the notification about the incorrect reading.

Our rheumatology colleagues also continued the ACTH biweekly injections because the rash had deteriorated. In addition, the patient received trastuzumab $100 \mathrm{mg}$ daily. At press time, she had received 7 cycles of chemotherapy, her most recent PET-CT scan after 16 months of starting the first treatment was showing stability of the disease, and she continued to have rash and areas of hyperpigmentation that did not progress.

\section{Conclusion}

The Bohan and Peter classification criteria for dermatomyositis include proximal muscle weakness in the presence or absence of pulmonary involvement; muscle biopsy evidence of inflammation; elevation of skeletal muscle enzymes; the

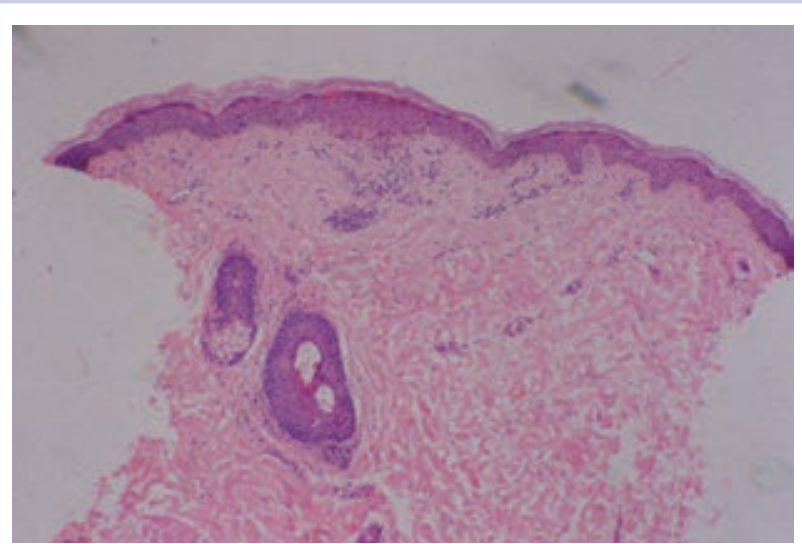

FIGURE 2 Dermatomyositis with concordant vacuolar interface dermatitis.

electromyographic triad of short, small, polyphasic motor units; and the dermatological features of rash of dermatomyositis. ${ }^{8}$ The characteristic cutaneous manifestations of dermatomyositis include Gottron papules, heliotrope eruption, photodistributed poikiloderma (including shawl and $\mathrm{V}$ signs), holster sign, calcinosis cutis, psoriasiform changes in the scalp, and generalized erythroderma, ${ }^{9}$ of which Gottron papules and heliotrope eruption are considered pathognomonic of dermatomyositis.

Dermatomyositis has been associated with cancer, and its incidence varies from less than $7 \%$ to more than $30 \%{ }^{4}$ The earliest reported malignancy associated with dermatomyositis was in 1916, when simultaneous occurrences of polymyositis and gastric carcinoma were reported. ${ }^{3}$ Breast cancer is the most common cancer in women in the United States, with a lifetime incidence of 1 in 8 women. In nearly $20 \%$ of cases of malignancy associated dermatomyositis, the underlying malignancy is breast cancer. ${ }^{5}$ Treatment of dermatomyositis is usually with glucocorticoids by mouth, and in severe cases, it is initiated with intravenous methylprednisolone. Glucocorticoidsparing agents such as methotrexate and azathioprine are used along with glucocorticoids. Azathioprine is used in patients who have interstitial lung disease associated with dermatomyositis. Response to treatment is followed by testing muscle strength on serial physical exams. While patients are on high-dose immunosuppressive agents, pneumocystis carinii pneumonia prophylaxis with TMPSMX (trimethoprim-sulfamethoxazole) is initiated. In cases of dermatomyositis-associated with malignancy, resolution of symptoms coincides with treatment of malignancy. ${ }^{10}$

Several cases of paraneoplastic dermatomyositis have been described in the literature. Most of them report an improvement of skin findings upon initiation of treatment for the underlying malignancy or the malignancy is treated. ${ }^{11}$ We were therefore surprised to notice a worsen- 
ing of our patient's skin rash even though there was no evidence of progression of underlying the underlying breast cancer malignancy on repeat PET-CT scan. As physicians, we should be increasingly aware of such uncommon presentations of paraneoplastic dermatomyositis and seek to find alternative therapies because prompt recognition and

\section{References}

1. Yuste-Chaves M, Unamuno-Perez P. Cutaneous manifestations of systemic malignancies: Part 2. Actas Dermosifiliogr. 2013;104:543-553.

2. Dalakas MC, Hohlfeld R. Polymyositis and dermatomyositis. Lancet. 2003;362:971-982.

3. Sandhu NP, Zakaria S, Degnim AC, Boughey JC. Dermatomyositis presenting as a paraneoplastic syndrome due to underlying breast cancer. BMJ Case Rep. doi: 10.1136/bcr.10.2010.3416. Published February 2, 2011. Accessed February 8, 2016.

4. Callen JP, Hyla JF, Bole GG Jr, et al. The relationship of dermatomyositis and polymyositis to internal malignancy. Arch Dermatol. 1980;116:295-298.

5. Barnes BE, Mawr B. Dermatomyositis and malignancy. A review of the literature. Ann Intern Med. 1976;84:68-76.

6. Airio A, Pukkala E, Isomaki H. Elevated cancer incidence in patients with dermatomyositis: a population based study. J appropriate treatment can significantly impact the quality of life for these patients.

\section{Acknowledgments}

The authors thank the patient for allowing them to share the details of her case.

Rheumatol. 1995;22:1300-1303.

7. Tian M, Zhong Y, Zhou F, Xie C, Zhou Y, Liao Z. Platinum-based therapy for triple-negative breast cancer treatment: a meta-analysis. Mol Clin Oncol. 2015:3:720-724.

8. Bohan A, Peter JB. Polymyositis and dermatomyositis: parts 1 and 2. N Engl J Med. 1975;292:344-347, 403-407.

9. Callen JP, Wortmann RL. Dermatomyositis. Clin Dermatol. 2006;24:363-373.

10. Joffe MM, Love LA, Leff RL, et al. Drug therapy of idiopathic imflammatory myopathies: predictor of response to prednisone, azathioprine, and methotrexate and a comparison of their efficacy. Am J Med. 1993;94:379-387.

11. Song YJ, Wu YF, Fan T. Dermatosis as the initial manifestation of malignant breast tumors: retrospective analysis of 4 cases. Breast Care (Basel). 2010;5:174-176. 